\title{
COREFLECTIVE SUBCATEGORIES
}

\author{
BY \\ HORST HERRLICH AND GEORGE E. STRECKER
}

\begin{abstract}
General morphism factorization criteria are used to investigate categorical reflections and coreflections, and in particular epi-reflections and monocoreflections. It is shown that for most categories with "reasonable" smallness and completeness conditions, each coreflection can be "split" into the composition of two mono-coreflections and that under these conditions mono-coreflective subcategories can be characterized as those which are closed under the formation of coproducts and extremal quotient objects. The relationship of reflectivity to closure under limits is investigated as well as coreflections in categories which have "enough" constant morphisms.
\end{abstract}

1. Introduction. The concept of reflections in categories (and likewise the dual notion-coreflections) serves the purpose of unifying various fundamental constructions in mathematics, via "universal" properties that each possesses. Historically, the concept seems to have its roots in the fundamental construction of E. Čech [4] whereby (using the fact that the class of compact spaces is productive and closed-hereditary) each completely regular $T_{2}$ space is densely embedded in a compact $T_{2}$ space with a universal extension property. In [3, Appendice III; Sur les applications universelles] Bourbaki has shown the essential underlying similarity that the Čech-Stone compactification has with other mathematical extensions, such as the completion of uniform spaces and the embedding of integral domains in their fields of fractions. In doing so, he essentially defined the notion of reflections in categories. It was not until 1964, when Freyd [5] published the first book dealing exclusively with the theory of categories, that sufficient categorical machinery and insight were developed to allow for a very simple formulation of the concept of reflections and for a basic investigation of reflections as entities themselves $\left({ }^{1}\right)$. The introduction of the categorical approach also automatically yielded the dual theory of coreflections which previously seems not to have been generally investigated $\left({ }^{2}\right)$. Since Freyd's beginning investigation, significant general results

Received by the editors August 29, 1967.

AMS 1969 subject classifications. Primary 1810; Secondary 0810.

Key words and phrases. Coreflective subcategory, mono-coreflection, reflective subcategory, epi-reflection, epi-mono factorization, extremal epimorphism, extremal monomorphism.

( $\left.{ }^{1}\right)$ The initial definition of reflection, and categorical results relating to it, actually first appeared in 1960 in Freyd's thesis, Functor theory.

${ }^{(2)}$ In 1963 Gleason [6] pointed out a type of construction that could be performed in the category of topological spaces, which generalized his construction that associated with each topological space a locally connected space having a universal mapping property. These methods and ideas seem to have been the forerunner of the general study of coreflective subcategories. Copyright (C) 1971, American Mathematical Society 
concerning reflective subcategories have been obtained by Isbell [14], [16], Kennison [18], Hušek [11], [12], and Baron [1], [2]. Some more specialized results, mainly within the realm of general topology but having implications in the theory of reflective and coreflective subcategories, have been obtained by Arhangelskiǐ, Franklin, Gleason, Isbell, Kennison, Steenrod, van der Slot, and the authors of this paper.

This is the first of two papers which will be concerned specifically with the notion of coreflections. The current paper, Coreflective subcategories will deal within the realm of general category theory and will provide, along with some new results about coreflective subcategories, some generalizations of theorems which are more or less well known to specialists in the field. Note that because of the intrinsic nature of category theory, each result in this paper has a corresponding dual statement of concomitant interest. For the sake of brevity, however, we will refrain from forming these dualizations.

The second paper, Coreflective subcategories in general topology will provide some applications of the general theory of coreflections to general topology. In doing so, similarities among classes of such seemingly dissimilar topological structures as discrete spaces, locally connected spaces, sequential spaces, and $k$-spaces will become apparent. It will be shown that many of the "nice" properties of these classes follow immediately from the fact that each class is a coreflective subcategory of the category of topological spaces. Attendant generalizations of some of these topological notions and of theorems valid for them will follow.

2. Preliminaries. In this section we state some necessary definitions, give some examples and provide several preliminary results concerning coreflective subcategories. Theorems 1 and 2 are two rather well-known results due to Freyd about the preservation of limits and colimits by coreflective subcategories. We include the proofs for the sake of completeness.

Assumptions. In what follows, each category under consideration will be assumed to have at least one object, and all subcategories will be assumed to be both full and replete $\left({ }^{3}\right)$. All categorical notions which are not defined explicitly will have the connotation ascribed to them by Mitchell $[19]\left({ }^{4}\right)$.

Definition 1. If $\mathfrak{A}$ is a subcategory of the category $\mathbb{E}$ and if for each object $X$ in $\mathfrak{E}$, there exists an object $X_{\mathfrak{U}}$ in $\mathfrak{A}$ and a morphism $c_{\mathfrak{U}}: X_{\mathfrak{A}} \rightarrow X$ such that for each object $A$ in $\mathfrak{A}$ and each morphism $f: A \rightarrow X$, there exists a unique morphism $f^{\prime}: A \rightarrow X_{\mathfrak{U}}$ such that the diagram

$\left({ }^{3}\right)$ A subcategory $\mathscr{U}$ of a category $\mathfrak{C}$ is said to be replete if each object in $\mathfrak{C}$ which is isomorphic to an object in $\mathfrak{A}$, must be in $\mathscr{A}$.

$\left.{ }^{4}\right)$ Note that the most crucial definition of this paper, i.e., that of a coreflection, is dual to that in [19]. The definition used here is standard with most authors, however. 


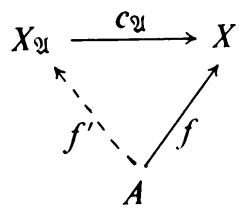

commutes, then $\mathfrak{A}$ is said to be a coreflective subcategory of $\mathfrak{C}$ (or is coreflective in $\mathfrak{E})$ and $c_{\mathfrak{U}}$ is called a coreflection morphism from $X_{\mathfrak{U}}$ to $X$. Furthermore, if each $c_{\mathfrak{U}}$ is a monomorphism (resp. an epimorphism) then $\mathfrak{U}$ is said to be a monocoreflective (resp. an epi-coreflective) subcategory of $\mathfrak{\mathfrak { C }}$.

The dual concepts are reflective subcategory and reflection morphism.

ExAmples. 1. The category of all torsion groups is mono-coreflective in the category of all abelian groups.

2. The category of all groups, each of which coincides with its commutator subgroup, is mono-coreflective in the category of all groups.

3. The category of all commutative nilrings is mono-coreflective in the category of all commutative rings.

4. The category of all fine uniform spaces is both mono-coreflective and epicoreflective in the category of all uniform spaces.

5 . The category of all locally connected spaces (resp. $k$-spaces, resp. sequential spaces) is both mono-coreflective and epi-coreflective in the category of all topological spaces $\left({ }^{5}\right)$.

LEMMA 1. If $\mathfrak{A}$ is a coreflective subcategory of the category $\mathfrak{E}$, with coreflection morphisms $c_{\mathfrak{A}}: X_{\mathfrak{A}} \rightarrow X$, and if $f$ and $g$ are morphisms in $\mathfrak{A}$ such that for some $c_{\mathfrak{A}}$, $c_{\mathfrak{A}} f=c_{\mathfrak{U}} g$, then $f=g$.

Proof. Immediate from the uniqueness requirement of Definition 1.

Proposition 1. Every epi-coreflective subcategory, $\mathfrak{A}$, of a category, $\mathfrak{\mathfrak { C }}$, is also mono-coreflective in $\mathfrak{C}$.

Proof. Let $c_{\mathfrak{A}}: X_{\mathfrak{U}} \rightarrow X$ be any coreflection morphism such that for given morphisms $f, g: Y \rightarrow X_{\mathfrak{U}}$ in $\mathfrak{E}, c_{\mathfrak{U}} f=c_{\mathfrak{U}} g$. Since $Y$ is in $\mathfrak{E}$ there exists a coreflection epimorphism $c_{\mathfrak{Q}}^{\prime}: Y_{\mathfrak{Q}} \rightarrow Y$. Now

$$
c_{\mathfrak{A}}\left(f c_{\mathfrak{Q}}^{\prime}\right)=\left(c_{\mathfrak{U}} f\right) c_{\mathfrak{U}}^{\prime}=\left(c_{\mathfrak{U}} g\right) c_{\mathfrak{U}}^{\prime}=c_{\mathfrak{U}}\left(g c_{\mathfrak{I}}^{\prime}\right) .
$$

Thus since $f c_{\mathfrak{A}}^{\prime}$ and $g c_{\mathfrak{U}}^{\prime}$ are in $\mathfrak{A}$, by Lemma 1 it follows that $f c_{\mathfrak{A}}^{\prime}=g c_{\mathfrak{U}}^{\prime}$ so that since $c_{\mathfrak{A}}^{\prime}$ is an epimorphism, $f=g$. Hence $c_{\mathfrak{U}}$ is a monomorphism.

Definition 2. Let $\mathbb{E}$ be a category and let $I$ be a small category.

(i) A diagram in $\mathfrak{E}$ over $I$ is a functor $D: I \rightarrow \mathfrak{C}$.

(5) A more detailed analysis of these topological constructions will be made in Coreflective subcategories in general topology. 
(ii) A lower bound of a diagram $D$ in $\mathfrak{E}$ over $I$ is a family $\left\{L \stackrel{q_{i}}{\rightarrow} D(i)\right\}$ (where $i$ runs through the objects of $I$ ) such that for each morphism $m: i \rightarrow j$ in $I$, the diagram

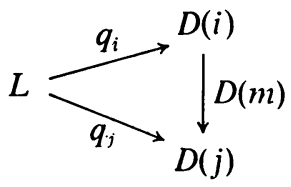

commutes.

(iii) A limit of a diagram $D$ in $\mathbb{E}$ over $I$ is a lower bound $\left\{L \stackrel{q_{i}}{\rightarrow} D(i)\right\}$ of $D$ such that for any lower bound $\left\{L^{\prime} \stackrel{q^{\prime}}{\rightarrow} D(i)\right\}$ of $D$, there exists a unique morphism $f: L^{\prime} \rightarrow L$ such that for each $i$ the diagram

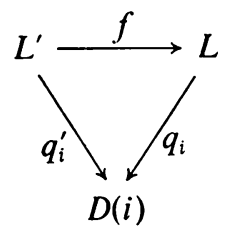

commutes.

Definition 3. A category $\Subset$ is said to be complete provided that for each small category, $I$, every diagram in $\mathbb{E}$ over $I$ has a limit.

Dual notions are upper bound of a diagram, colimit of a diagram, and cocomplete.

THEOREM 1 (FREYD). Let I be a small category and $\mathfrak{A}$ be a coreflective subcategory of a category $\mathfrak{E}$. If a diagram $D$ in $\mathfrak{A}$ over I has a colimit $\left\{D(i) \stackrel{q_{i}}{\rightarrow} L\right\}$ in $\mathfrak{E}$, then $L$ is in $\mathfrak{A}$.

Proof. Let $c_{\mathfrak{U}}: L_{\mathfrak{A}} \rightarrow L$ be the coreflection morphism for $L$. Then for each $i$ in $I$ there exists a morphism $q_{i}^{\prime}: D(i) \rightarrow L_{\mathfrak{A}}$ such that $c_{\mathfrak{A}} q_{i}^{\prime}=q_{i}$. Thus for any morphism $m: i \rightarrow j$ in $I$, the diagram

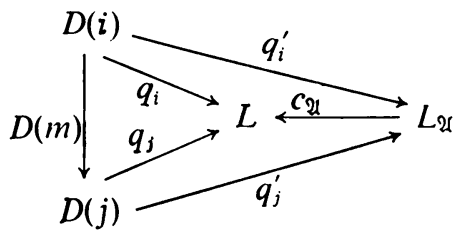

commutes. Hence $\left\{D(i) \stackrel{q_{\mathfrak{i}}}{\rightarrow} L_{\mathfrak{A}}\right\}$ is an upper bound of $D$ in $\mathbb{E}$ over $I$, so that there exists a morphism $f: L \rightarrow L_{\mathfrak{A}}$ such that for each $i$ in $I, f q_{i}=q_{i}^{\prime}$. Therefore

$$
\left(c_{\mathfrak{U}} f\right) q_{i}=c_{\mathfrak{A}}\left(f q_{i}\right)=c_{\mathfrak{A}} q_{i}^{\prime}=q_{i}=1_{L} q_{i}
$$


for each $i$ in $I$, so that by the uniqueness condition in the definition of colimit,

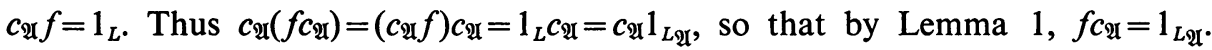
Hence $c_{\mathfrak{U}}$ is an isomorphism, so that since $\mathfrak{A}$ is replete in $\mathfrak{E}, L$ is in $\mathfrak{A}$.

COROLlaRY 1. Each coreflective subcategory of a cocomplete category is itself cocomplete and is a cocomplete subcategory.

COROLLARY 2. If $\mathfrak{C}$ is a category which has coproducts and if $\mathfrak{A}$ is a coreflective subcategory of $\mathfrak{C}$, then $\mathfrak{A}$ is closed under the formation of coproducts.

THEOREM 2 (FREYD). Let I be a small category and let $\mathfrak{A}$ be a coreflective subcategory of a category $\mathfrak{E}$, with coreflection morphisms $c_{\mathfrak{N}}: X_{\mathfrak{U}} \rightarrow X$. If a diagram $D$ in $\mathfrak{A}$ over I has a limit $\left\{L \stackrel{q_{i}}{\longrightarrow} D(i)\right\}$ in $\mathfrak{E}$, then it has the limit $\left\{L_{\mathfrak{Q}} \stackrel{\mathfrak{C}_{\mathfrak{U}}}{\longrightarrow} L \stackrel{\mathfrak{q}_{\mathfrak{i}}}{\longrightarrow} D(i)\right\}$ in $\mathfrak{A}$.

Proof. Clearly $\left\{L_{\mathfrak{Q}} \stackrel{q_{i} \mathfrak{C}}{\longrightarrow} D(i)\right\}$ is a lower bound of $D$ in $\mathfrak{A}$ over $I$. Suppose that $\left\{A \stackrel{r_{i}}{\rightarrow} D(i)\right\}$ is also a lower bound of $D$ in $\mathfrak{A}$ over $I$. Since $\left\{L \stackrel{q_{t}}{\rightarrow} D(i)\right\}$ is a limit, there exists a unique morphism $f: A \rightarrow L$ such that for each $i$ in $I, r_{i}=q_{i} f$. Thus since $c_{\mathscr{V}}$ is a coreflection, there exists a unique morphism $f^{\prime}: A \rightarrow L_{\mathfrak{Q}}$ such that for each $i$ in $I$, the diagram

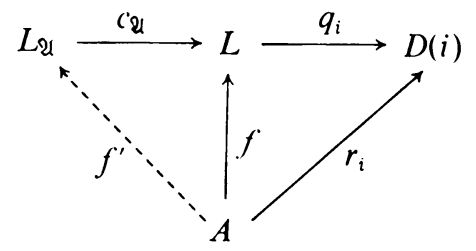

commutes. Hence $\left\{L_{\mathfrak{A}} \stackrel{q_{\mathfrak{l}} \mathfrak{C A}}{\longrightarrow} D(i)\right\}$ is a limit of $D$ in $\mathfrak{A}$ over $I$.

COROLlaRY. Each coreflective subcategory of a complete category is itself complete (but is not necessarily a complete subcategory).

Definition 4. Let $\mathbb{E}$ be a category.

(i) A morphism $f: A \rightarrow B$ in $\mathbb{C}$ is called an extremal epimorphism (or an extremal quotient of $A$ ) provided that the following conditions are satisfied:

(1) $f$ is an epimorphism.

(2) If for some morphism $h$ and some monomorphism $g, f=g h$; then $g$ is an isomorphism.

(ii) An object, $B$, is said to be an extremal quotient object of $A$ provided that there exists an extremal epimorphism $f: A \rightarrow B$.

Dual notions are extremal monomorphism and extremal subobject.

Notation. $\rightarrow$ and $\rightarrow$ will denote epimorphisms and extremal epimorphisms, respectively, while $\longleftrightarrow$ and $\gg$ will denote monomorphisms and extremal monomorphisms. 
REMARK. The notions of extremal epimorphism and extremal monomorphism are due to Isbell [15]. They are important concepts for several reasons.

1. For abelian categories, they are exactly the epimorphisms and monomorphisms, respectively. More generally, balanced categories can be characterized as those categories for which the epimorphisms are precisely the extremal epimorphisms or as those for which the monomorphisms are precisely the extremal monomorphisms.

2. In general categories, extremal epimorphisms lie in strength between the retractions and the epimorphisms, but still have an important property of the retractions, namely, a morphism is simultaneously an extremal epimorphism and a monomorphism if and only if it is an isomorphism. The dual statements are valid for extremal monomorphisms.

3. In particular categories for which the notions of retractions and coretractions are so restrictive that they are hard to work with, and the notions of epimorphism and monomorphism are too weak to have much significance, the extremal epimorphisms and extremal monomorphisms seem to be the mappings with real categorical relevance. For example, in the category of topological spaces and continuous functions, the retractions are the topological retractions, the epimorphisms are the onto maps, and the extremal epimorphisms are the quotient maps; the coretractions are topological embeddings whose images are retracts in the range, the monomorphisms are the one-to-one maps, and the extremal monomorphisms are the topological embeddings.

4. Extremal epimorphisms and extremal monomorphisms have special relevance to the theory of coreflections and reflections, as will be seen throughout the remainder of this paper $\left({ }^{6}\right)$.

One disadvantage connected with these morphisms is that, in general, there is no guarantee that the composition of extremal epimorphisms is an extremal epimorphism, or dually, that extremal monomorphisms are closed under compositions. We shall see below, however, (Proposition 5) that for categories satisfying a certain "morphism lifting" property (which are actually the categories of interest to us) these compositions are well behaved( $\left.{ }^{7}\right)$.

Proposition 2. In any category, if $e: X \rightarrow Y$ is an equalizer for morphisms $f, g: Y \rightarrow W$, then $e$ is an extremal monomorphism.

$\left.{ }^{(}\right)$There are other types of epi(mono)morphisms, such as the special epi(mono)morphisms of Hilton [10], which can assume the role of extremal epi(mono)morphisms throughout much of the remainder of the paper. Indeed most of the following results can be reformulated in terms of bicategorical structures (cf. [13] and [18]). However, to do so would not significantly increase the generality of the results, and would in some cases decrease their generality.

$\left({ }^{7}\right)$ Other conditions which guarantee the preservation of these properties under compositions are given in [15]. 
Proof. First, $e$ must be a monomorphism since if $h, k: U \rightarrow X$ are morphisms such that $e h=e k$, then

$$
f(e k)=f(e h)=(f e) h=(g e) h=g(e h)=g(e k),
$$

so that by the definition of equalizer there is a unique morphism $l: U \rightarrow X$ such that $e l=e k=e h$. Thus $l=k=h$.

To show that $e$ is extremal, suppose that $e=r s$ where $s: X \rightarrow Z$ is an epimorphism.

$$
(f r) s=f(r s)=f e=g e=g(r s)=(g r) s .
$$

Thus since $s$ is an epimorphism, $f r=g r$, so that there exists a morphism $t$ such that $e t=r$. Now $e(t s)=(e t) s=r s=e=e 1_{X}$, so that since $e$ is a monomorphism, $t s=1_{X}$. Hence $s$ is a coretraction and an epimorphism, so it is an isomorphism.

Proposition 3. Every coreflective subcategory of any category is closed under the formation of retracts.

Proof. Suppose that $\mathfrak{A}$ is a coreflective subcategory of $\mathfrak{E}, A$ is an object of $\mathfrak{A}$ and $g: A \rightarrow X$ is a retraction in $\mathfrak{E}$. Let $c_{\mathfrak{U}}: X_{\mathfrak{A}} \rightarrow X$ be the coreflection morphism for $X$ and let $f: X \rightarrow A$ be a morphism such that $g f=1_{X}$. By the definition of coreflection there exists a morphism $g^{\prime}: A \rightarrow X_{\mathfrak{U}}$ such that the diagram

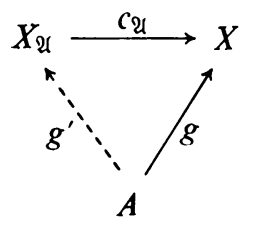

commutes. Now $c_{\mathfrak{U}}\left(g^{\prime} f\right)=\left(c_{\mathfrak{A}} g^{\prime}\right) f=g f=1_{X}$. Also each of $1_{X_{\mathfrak{M}}}$ and $\left(g^{\prime} f\right) c_{\mathfrak{A}}$ makes the diagram

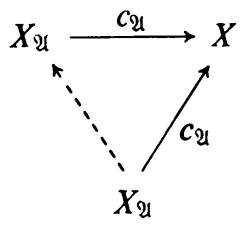

commute, so that by Lemma $1,\left(g^{\prime} f\right) c_{\mathfrak{A}}=1_{X_{\mathfrak{V}}}$. Hence $c_{\mathfrak{Q}}$ is an isomorphism, so that $X$ is an object of $\mathfrak{A}$.

Proposition 4. Every mono-coreflective subcategory of any category is closed under the formation of extremal quotients.

Proof. Let $\mathfrak{A}$ be mono-coreflective in $\mathfrak{E}, A$ an object of $\mathfrak{A}, f: A \rightarrow X$ an extremal epimorphism and $c_{\mathfrak{A}}: X_{\mathfrak{A}} \rightarrow X$ the coreflection morphism for $X$. By Definition 1, there exists some $f^{\prime}: A \rightarrow X_{\mathfrak{U}}$ such that $c_{\mathfrak{U}} f^{\prime}=f$; but since $c_{\mathfrak{U}}$ is a monomorphism, and $f$ is an extremal epimorphism, $c_{\mathfrak{A}}$ must be an isomorphism. 


\section{Factorization properties.}

Definition 5. A morphism $f$ in a category $\mathbb{C}$ is said to have

(i) an (extremal) epi-mono factorization provided that for some (extremal) epimorphism $g$ in $\mathbb{E}$ and some monomorphism $h$ in $\mathfrak{E}, f=h g$;

(ii) a unique extremal epi-mono factorization provided that it has an extremal epimono factorization $f=h g$ and if $f=h^{\prime} g^{\prime}$ is an extremal epi-mono factorization, then there exists an isomorphism $u$ in $\mathbb{E}$, such that the diagram

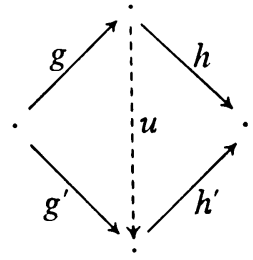

commutes.

Definition 6. A category cs is said to have

(i) the (extremal) epi-mono factorization property provided that each of its morphisms has an (extremal) epi-mono factorization;

(ii) the unique extremal epi-mono factorization property provided that each of its morphisms has a unique extremal epi-mono factorization;

(iii) the strong unique extremal epi-mono factorization property provided that it has the unique extremal epi-mono factorization property and, in $\Subset$, the composition of extremal epimorphisms is an extremal epimorphism.

Dual notions are: (strong) (unique) epi-(extremal) mono factorization property.

REMARK. Although at first glance it may seem to be a fairly stringent requirement, Theorem 3, below, and its corollary show that most categories which are frequently studied have both the unique extremal epi-mono factorization property and the unique epi-extremal mono factorization property. Consequently since the requirement that the composition of extremal epimorphisms be an extremal epimorphism and that the composition of extremal monomorphisms be an extremal monomorphism is not very restrictive, many of the familiar categories have both the strong unique extremal epi-mono factorization property and the strong unique epi-extremal mono factorization property.

THEOREM 3. Let $\mathfrak{c}$ be a category which

(i) has products,

(ii) has the epi-mono factorization property,

(iii) is either locally small or colocally small;

then $\mathbb{C}$ has the unique extremal epi-mono factorization property.

Proof. Let $f: X \rightarrow Y$ be a morphism in $\mathfrak{c}$. We wish to find a unique extremal epi-mono factorization for $f$. By (iii) there exists a set $\left\{X \stackrel{e_{i}}{\rightarrow} S_{i} \stackrel{m_{i}}{\longrightarrow} Y, i \in I\right\}$ such 
that each epi-mono factorization of $f$ is (up to isomorphism) of the form $f=m_{i} e_{i}$ for some $i$. Let $\left\{P \stackrel{p_{i}}{\rightarrow} S_{i}\right\}$ be a product of the family $\left\{S_{i}, i \in I\right\}$. There exists a morphism $g: X \rightarrow P$ such that for each $i \in I, p_{i} g=e_{i}$. Let $g=m e$ be an epi-mono factorization for $g$. Thus for each $i$, the diagram

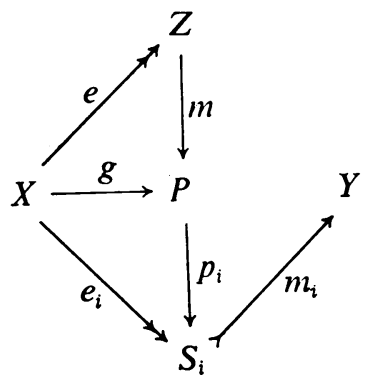

commutes.

Now for each $i, j \in I$,

$$
\left(m_{i} p_{i} m\right) e=m_{i}\left(p_{i} g\right)=m_{i} e_{i}=f=m_{j} e_{j}=m_{j}\left(p_{j} g\right)=\left(m_{j} p_{j} m\right) e .
$$

Therefore since $e$ is an epimorphism, $m_{i} p_{i} m=m_{j} p_{j} m$ for all $i, j \in I$. Let us call this morphism $q$.

(1) $q$ is a monomorphism.

Let $h$ and $k$ be morphisms such that $q h=q k$. Then for each $i \in I, m_{i} p_{i} m h=q h=$ $q k=m_{i} p_{i} m k$, so that since $m_{i}$ is a monomorphism $p_{i} m h=p_{i} m k$. Thus $m h=m k$, and since $m$ is a monomorphism, $h=k$. Thus we have that $f=q e$ is an epi-mono factorization for $f$, so that we may assume that there is some $k \in I$ such that (up to isomorphism) $e=e_{k}$ and $q=m_{k}$.

(2) $e=e_{k}$ is an extremal epimorphism.

Let $e=u v$ be a factorization of $e_{k}$ where $u$ is a monomorphism, and let $v=r s$ be an epi-mono factorization of $v$. Then $\left(m_{k} u r\right) s$ is an epi-mono factorization of $f$, so that there exists some $i \in I$ and isomorphism $t: S_{i} \rightarrow V$ such that the following diagram commutes.

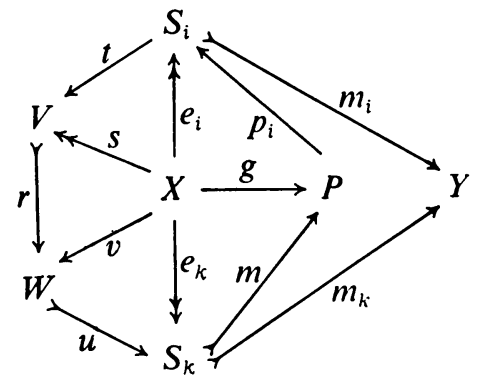

Now consider the morphism $w=r t p_{i} m$.

$$
m_{k}(u w)=\left(m_{k} u r t\right) p_{i} m=m_{i} p_{i} m=m_{k}=m_{k} 1_{S_{k}} .
$$


Since $m_{k}$ is a monomorphism, we have $u w=1_{S_{k}}$. Thus $u$ is a retraction. Since it is a monomorphism, it must be an isomorphism.

(3) The extremal epi-mono factorization $f=m_{k} e_{k}$ is unique.

Suppose that there is some other extremal epi-mono factorization for $f$. By (iii) it has (up to isomorphism) the form $f=m_{j} e_{j}$ for some $j \in I$. We wish to show that $p_{j} m$ is an isomorphism.

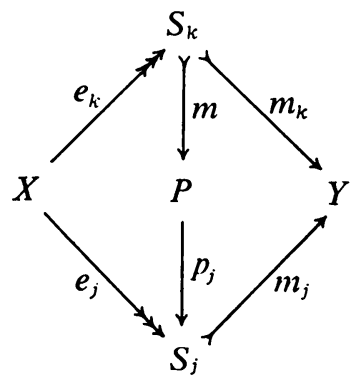

Let $g^{\prime}$ and $h^{\prime}$ be morphisms such that $\left(p_{j} m\right) g^{\prime}=\left(p_{j} m\right) h^{\prime}$. Then $m_{k} g^{\prime}=\left(m_{j} p_{j} m\right) g^{\prime}$ $=\left(m_{j} p_{j} m\right) h^{\prime}=m_{k} h^{\prime}$; so that since $m_{k}$ is a monomorphism it follows that $g^{\prime}=h^{\prime}$. Thus $p_{j} m$ is a monomorphism. But $\left(p_{j} m\right) e_{k}$ is a factorization of $e_{j}$, which is an extremal epimorphism. Hence $p_{j} m$ is an isomorphism.

COROLlaRY. If $\mathfrak{E}$ is a category which has products and coproducts, is epi-mono factorable, and is either locally small or colocally small, then $₫$ has both the unique extremal epi-mono factorization property and the unique epi-extremal mono factorization property.

REMARK. The following proposition gives a characterization of the strong unique extremal epi-mono factorization property in terms of a certain diagram diagonalizing property.

Proposition 5. If $\mathbb{E}$ is a category which has the extremal epi-mono factorization property, then the following are equivalent:

(1) $\mathbb{C}$ has the strong unique extremal epi-mono factorization property.

(2) For each commutative diagram in $\mathbb{E}$ of the form

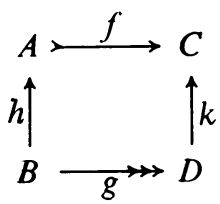

there exists a unique morphism $e: D \rightarrow A$ such that the diagram

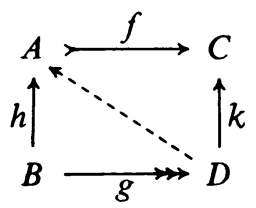

commutes. 
Proof. [(1) $\Rightarrow(2)]$. By factoring each of $h$ and $k$, we obtain

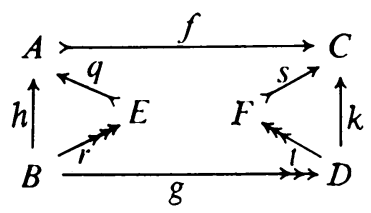

Then $(f q) r$ and $s(t g)$ are each extremal epi-mono factorizations of $f h$. Thus there exists an isomorphism $v: F \rightarrow E$ such that $s=f q v$ and $r=v t g$. Then $e=q v t$ is the required morphism.

$[(2) \Rightarrow(1)]$. Suppose that $r s=t u$ are each extremal epi-mono factorizations. By (2) there exist morphisms $v$ and $w$ such that the diagrams
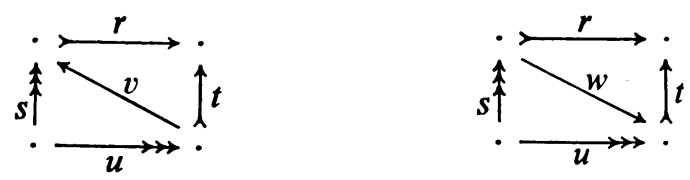

commute. Thus, $(v w) s=v(w s)=v u=s$ and $t(w v)=(t w) v=r v=t$. Hence since $s$ is an epimorphism and $t$ is a monomorphism, $v$ is an isomorphism. Therefore $\mathbb{E}$ has the unique extremal epi-mono factorization property.

To show that the property is "strong", let $f$ and $g$ be extremal epimorphisms such that the composition $g f$ is defined and such that $g f=k h$ where $k$ is a monomorphism. Making use of (2) twice, we obtain the existence of morphisms $p$ and $q$ which make the diagram

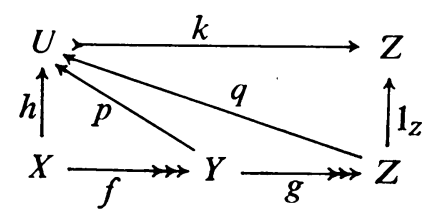

commute. Thus $k$ is both a monomorphism and a retraction, so that it is an isomorphism; which shows that $g f$ is an extremal epimorphism.

4. Mono-coreflections vs. coreflections. The list of examples in $\$ 2$ tends to lead one to believe that the study of coreflections is general might not be seriously handicapped if one studied only mono-coreflective subcategories. In this section we will show some more reasons for the emphasis on mono-coreflections. Theorem 4, which generalizes a theorem stated in the dual by Kennison [18] shows that for categories with "reasonable" properties, each coreflection has a categorical splitting into two mono-coreflections. The generalization seems to be significant since there is no requirement that the categories under consideration be locally small, colocally small, have pushouts or be closed under products or coproducts as is the case in [18].

This natural emphasis on mono-coreflections as opposed to coreflections in general is also not particularly surprising since, in the dual case, nearly every well- 
known example of a reflective subcategory is epi-reflective. However, most wellknown examples of reflections are not mono-reflections, so that it is surprising that in so many standard categories (e.g. topological spaces, uniform spaces, and ordered sets) all coreflections are epi-coreflections (and so by Proposition 1 are also monocoreflections). Theorem 5 below shows that the reason for this is that for these categories, there exist "enough" constant morphisms.

Theorem 4 (SplitTing TheORem). Let $\mathfrak{c}$ be a category with the strong unique extremal epi-mono factorization property. Let $\mathfrak{A}$ be a coreflective subcategory of $\mathfrak{E}$ with coreflection morphisms $c_{\mathfrak{A}}: X_{\mathfrak{A}} \rightarrow X$. Then if $\mathfrak{B}$ is the subcategory of $\mathfrak{\mathfrak { C }}$ composed of all extremal quotient objects of objects in $\mathfrak{A}$, the following statements are valid:

(1) $\mathfrak{B}$ is mono-coreflective in $\mathfrak{E}$. (We denote the coreflection morphisms by $d_{\mathfrak{B}}: X_{\mathfrak{B}} \rightarrow X$.)

(2) $\mathfrak{A}$ is both epi-coreflective in $\mathfrak{B}$ and mono-coreflective in $\mathfrak{B}$; in fact, the coreflection morphisms $b_{\mathfrak{U}}: B_{\mathfrak{A}} \rightarrow B$ are even extremal epimorphisms in $\mathfrak{\mathbb { V }}$.

(3) For each $X$ in $\mathbb{E}$ the diagram

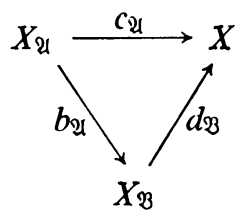

gives the unique extremal epi-mono factorization of $c_{\mathfrak{U}}$ in $\mathfrak{\mathbb { S }}$.

(4) $\mathfrak{A}=\mathfrak{B}$ provided that $\mathfrak{A}$ is mono-coreflective in $\mathfrak{\mathfrak { S }}$.

Proof of (1). Let $X$ be an object of $\mathfrak{C}$. The coreflection $c_{\mathfrak{A}}: X_{\mathfrak{U}} \rightarrow X$ has an extremal epi-mono factorization

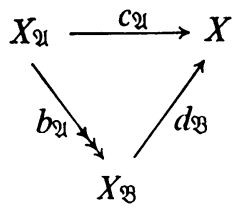

By the definition of $\mathfrak{B}, X_{\mathfrak{B}}$ is an object of $\mathfrak{B}$. We wish to show that $d_{\mathfrak{B}}$ is a coreflection for $\mathfrak{B}$.

Assume that $B$ is in $\mathfrak{B}$ and $f: B \rightarrow X$. By the definition of $\mathfrak{B}$, there exists some $A$ in $\mathfrak{A}$ and some extremal epimorphism $g: A \rightarrow B$. Now since $c_{\mathfrak{U}}$ is a coreflection and $f g: A \rightarrow X$, there exists a unique morphism $k: A \rightarrow X_{\mathfrak{A}}$ such that the diagram

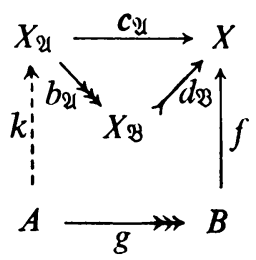


commutes. According to Proposition 5 there exists a unique $f^{\prime}$ such that the diagram

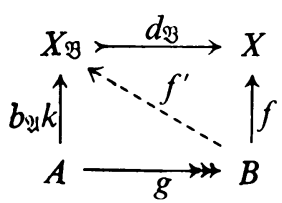

commutes. Hence $\mathfrak{B}$ is mono-coreflective in $\mathfrak{C}$.

Proof of (2) and (3). Let $B$ be an object of $\mathfrak{B}$. Since $B$ is an object of $\mathfrak{C}$, there exists a coreflection morphism $c_{\mathfrak{U}}: X_{\mathfrak{U}} \rightarrow B$. To show that $c_{\mathfrak{V}}$ is an extremal epimorphism in $\mathfrak{E}$, let

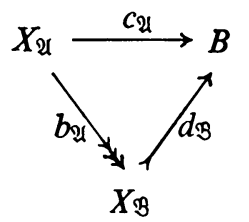

be the extremal epi-mono factorization of $c_{\mathfrak{A}}$. By (1), $d_{\mathfrak{B}}$ is a coreflection morphism so that there exists a morphism $f$ such that the diagram

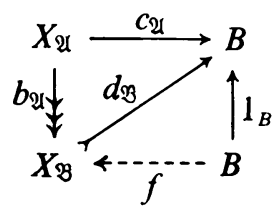

commutes. Thus $d_{\mathfrak{B}}$ is a retraction and a monomorphism, so it is an isomorphism. Hence $c_{\mathfrak{U}}$ is an extremal epimorphism in $\mathfrak{E}$. By Proposition $1, b_{\mathfrak{A}}$ is also a monomorphism in $\mathfrak{B}$. (3) follows immediately from this construction.

Proof of (4). If $\mathfrak{A}=\mathfrak{B}$, by (1), $\mathfrak{A}$ is mono-coreflective in $\mathfrak{E}$. The converse follows from Proposition 4.

Definition 7. A morphism $f: X \rightarrow Y$ in a category, $\mathfrak{E}$, is called constant provided that for each object $Z$ in $\subseteq$ and for each pair of morphisms $g, h: Z \rightarrow X$, it follows that $f g=f h$.

Definition 8. A category $\mathfrak{E}$ is said to be constant-generated provided that for each pair of objects $(X, Y)$ in $\mathbb{S}$

(i) the set of morphisms from $X$ to $Y$ is nonempty,

(ii) if $f, g: X \rightarrow Y$ are distinct morphisms, then there exists an object $Z$ and a constant morphism $k: Z \rightarrow X$ such that $g k$ and $f k$ are distinct.

Dual notions are coconstant morphisms and coconstant-generated categories.

REMARK. Many natural, often used categories are constant-generated; for example, the categories of nonempty sets, nonempty ordered sets, nonempty topological spaces, nonempty Hausdorff spaces, and nonempty uniform spaces. However, there is a paucity of well-known categories which are coconstant- 
generated. The category of all Boolean algebras is coconstant-generated. In that category the coconstant morphisms are precisely the 2-valued homomorphisms.

LEMMA 2. In any category, if $f: X \rightarrow X$ is a morphism and $g: X \rightarrow Y$ is a constant morphism, then $g f=g$.

LEMMA 3. In any category, if $f, g: X \rightarrow Y$ are constant morphisms, $h: Z \rightarrow X$ is a morphism and the set of morphisms from $X$ to $Z$ is nonempty, then $f h=g h$ implies that $f=g$.

Proof. Let $k: X \rightarrow Z$ be a morphism. By Lemma $2, f=f(h k)=(f h) k=(g h) k$ $=g(h k)=g$.

THEOREM 5. If $\mathfrak{A}$ is a coreflective subcategory of a constant-generated category, $\mathfrak{\mathfrak { S }}$, then $\mathfrak{A}$ is both mono-coreflective and epi-coreflective in $\mathfrak{\mathfrak { C }}$.

Proof. Let $X$ be an object of $\mathfrak{E}$ and let $c_{\mathfrak{A}}: X_{\mathfrak{A}} \rightarrow X$ be the coreflection morphism. By Proposition 1, we need only show that $c_{\mathfrak{A}}$ is an epimorphism.

Suppose that $f, g: X \rightarrow Y$ are distinct morphisms. By Definition 8, there exists a constant morphism $k: Z \rightarrow X$ such that $f k \neq g k$. Clearly $f k$ and $g k$ are constant morphisms. Let $A$ be an object of $\mathfrak{A}$, and let $h: A \rightarrow Z$ be arbitrary. Then by Lemma $3, f k h \neq g k h$. Now $c_{\mathfrak{U}}$ is a coreflection morphism so that there exists a morphism $s: A \rightarrow X_{\mathfrak{U}}$ such that the diagram

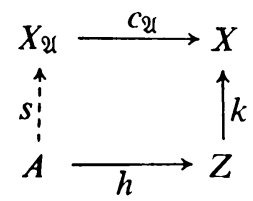

commutes. Thus $\left(f c_{\mathfrak{U}}\right) s=f(k h) \neq g(k h)=\left(g c_{\mathfrak{H}}\right) s$, so that $f c_{\mathfrak{U}} \neq g c_{\mathfrak{U}}$. Hence $c_{\mathfrak{U}}$ is an epimorphism.

RemarK. Additional results about constant morphisms in categories and about their relationship to coreflections can be found in [8]. For example: Each leftclosed subcategory of a factorable, locally small, pointed category, $\mathfrak{}$, with coproducts, is mono-coreflective in $\mathbb{E}$.

5. Characterizations of mono-coreflections. The last section has shown some reasons for the importance of mono-coreflections. This section contains two general theorems which characterize them. The first goes back to a theorem of Freyd [5, p. 87] which has been improved by Isbell [14], Kennison [18], and Baron [1], [2]. Kennison [17], van der Slot [9] and the first author of this paper [7], [9], also investigated the dual theorem (i.e., the characterization of epi-reflections) for the special case of categories of topological spaces. The current version (Theorem 6) further refines the previous ones. The second characterization theorem (Theorem 8) generalizes a similar theorem of the first author of this paper [7, Theorem 1.21] which dealt especially with categories of topological spaces. 
THEOREM 6. If $\mathfrak{E}$ is a category which

(i) is locally small,

(ii) has coproducts,

(iii) has the extremal epi-mono factorization property;

and if $\mathfrak{A}$ is a subcategory of $\mathfrak{E}$, then the following statements are equivalent:

(1) $\mathfrak{A}$ is mono-coreflective in $\mathfrak{\mho}$.

(2) $\mathfrak{A}$ is closed under the formation of coproducts and extremal quotient objects.

Proof. The second corollary to Theorem 1 and Proposition 4 show that (1) implies (2). To show that (2) implies (1), let $X$ be an object of $\mathfrak{E}$ and let $\left\{A_{i} \stackrel{g_{i}}{\longrightarrow} X\right\}$ be a representative class of all subobjects of $X$ which belong to $\mathfrak{A}$. By (i) this class is a set, so that by (2) it has a coproduct $\left\{A_{i} \stackrel{u_{i}}{\rightarrow} A\right\}$ in $\mathfrak{A}$. By the definition of coproduct, there exists a morphism $g: A \rightarrow X$ such that for each $i$

commutes. Let
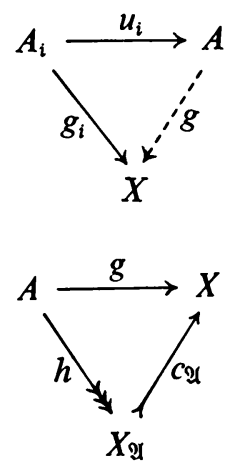

be the extremal epi-mono factorization of $g$. By (2) $X_{\mathfrak{U}}$ is in $\mathfrak{A}$. We wish to show that $c_{\mathfrak{U}}$ is a coreflection morphism.

Let $A^{\prime}$ be any object of $\mathfrak{A}, f: A^{\prime} \rightarrow X$ an arbitrary morphism and

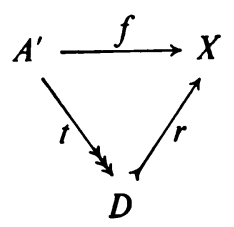

an extremal epi-mono factorization of $f$. Thus by (2) $D$ is in $\mathfrak{A}$, so that since $\left\{A_{i} \stackrel{g_{i}}{\longrightarrow} X\right\}$ is a representative class of all $\mathfrak{A}$-subobjects of $X$, we can assume that $D \stackrel{r}{\longrightarrow} X$ is the same as $A_{j} \stackrel{g_{g}}{\longrightarrow} X$ for some $j$. Thus we have the following commutative diagram:

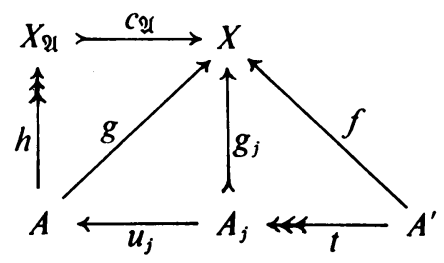


Let $f^{\prime}=h u_{j} t$. Then $f^{\prime}: A^{\prime} \rightarrow X_{\mathfrak{I}}$ and $c_{\mathfrak{A}} f^{\prime}=c_{\mathfrak{A}}\left(h u_{j} t\right)=\left(g u_{j}\right) t=g_{j} t=f$. The uniqueness of $f^{\prime}$ follows from the fact that $c_{\mathfrak{A}}$ is a monomorphism. Thus $c_{\mathfrak{A}}$ is a coreflection morphism.

Definition 9. Let $I$ be a small category, $\mathfrak{c}$ a category, $\mathfrak{A}$ a subcategory of $\mathfrak{c}$, and $D: I \rightarrow \mathfrak{E}$ a diagram in $\mathfrak{E}$ over $I$. $D$ is said to be partially in $\mathfrak{A}$ provided that for every object $i$ in $I$, there exists an object $j$ in $I$ such that $D(j)$ is in $\mathfrak{A}$ and there is some morphism $m: j \rightarrow i$.

Definition 10. A subcategory $\mathfrak{A}$ of a category $\mathfrak{C}$ is said to be strongly closed under limits in $\mathbb{E}$ provided that each limit in $\mathfrak{E}$ of every diagram which is partially in $\mathfrak{A}$ is itself in $\mathfrak{A}$. This definition may be restricted to a special type of limit such as equalizers or pullbacks, in which case we would say that $\mathfrak{A}$ is strongly closed under equalizers or strongly closed under pullbacks. For example, $\mathfrak{A}$ is strongly closed under pullbacks if and only if for each pullback diagram

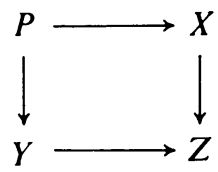

in $\mathfrak{E}$ with $X$ and $Y$ in $\mathfrak{A}$, it follows that $P$ is in $\mathfrak{A}$.

REMARK. Although it makes no difference from a categorical point of view, for the sake of convenience, we will state and prove the following two theorems in the language of the dual realm of reflective subcategories. The first theorem is related to Theorem 1.

THEOREM 7. If $\mathfrak{A}$ is an epi-reflective subcategory of a category $\mathfrak{E}$, then $\mathfrak{A}$ is strongly closed under limits in $\mathfrak{E}$.

Proof. (1) Let $D: I \rightarrow \mathfrak{C}$ be a diagram in $\mathfrak{夭}$ over $I$ which is partially in $\mathfrak{A}$. Let $\left\{L \stackrel{q_{\mathfrak{i}}}{\rightarrow} D(i)\right\}$ be a limit of $D$, let $J=\{i \in I \mid D(i) \in \mathfrak{A}\}$, and let $r_{\mathfrak{A}}: L \rightarrow L_{\mathfrak{A}}$ denote the reflection epimorphism. Thus for each $j$ in $J$ there exists a unique $q_{j}^{\prime}: L_{\mathfrak{A}} \rightarrow D(j)$ such that the diagram

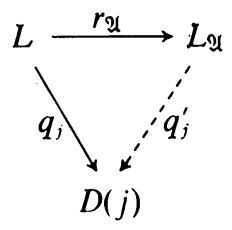

commutes.

(2) Now for each $i$ in $I$, there exists some $\hat{\imath}$ in $J$ and some $m_{i}: \hat{\imath} \rightarrow i$. For each $i$ in $I$, let $s_{i}=D\left(m_{i}\right)$ and define $t_{i}=s_{i} q_{i}^{\prime}: L_{\mathfrak{A}} \rightarrow D(i)$.

(3) $\left\{L_{\mathfrak{A}} \stackrel{t_{\mathfrak{t}}}{\rightarrow} D(i)\right\}$ is a lower bound of $D$ in (5 over $I$; for since $\left\{L \stackrel{q_{i}}{\rightarrow} D(i)\right\}$ is a limit, for each morphism $m: i \rightarrow j$ in $I$, all of the indicated triangles of the following diagram are commutative 


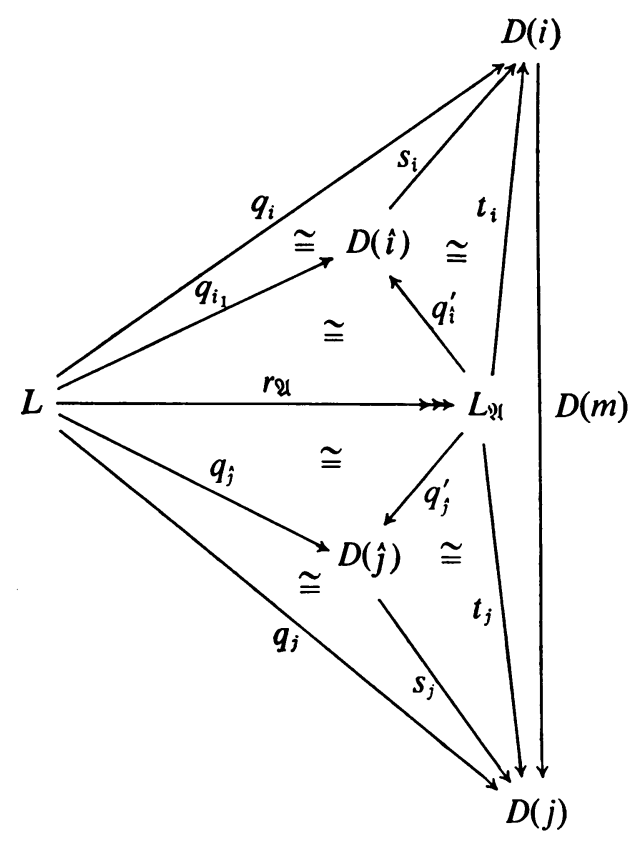

and $D(m) q_{i}=q_{j}$. Thus we have $D(m) t_{i} r_{\mathfrak{U}}=D(m) q_{i}=q_{j}=t_{j} r_{\mathfrak{A}}$, and since $r_{\mathfrak{U}}$ is an epimorphism, $D(m) t_{i}=t_{j}$.

(4) By (3) and the definition of limit there exists a morphism $h: L_{\mathfrak{A}} \rightarrow L$ such that for each $i, q_{i} h=t_{i}$. Thus for each $i$, the diagram

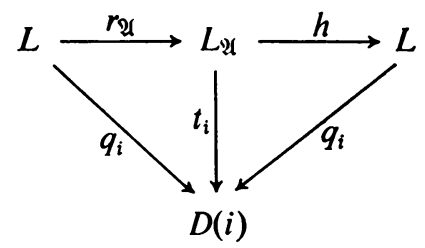

commutes; so that each of $1_{L}$ and $h r_{\mathfrak{A}}$ is a morphism $f: L \rightarrow L$ such that for each $i, q_{i} f=q_{i}$. Hence by the uniqueness requirement in Definition 2(iii), $1_{L}=h r_{\mathfrak{A}}$. Consequently, $r_{\mathfrak{A}}$ is an epimorphism and a coretraction, so that it is an isomorphism. Thus $L$ is in $\mathfrak{A}$.

THEOREM 8. Let 〔 be a category which

(i) is complete,

(ii) is colocally small,

(iii) has the epi-extremal mono factorization property,

(iv) has the property that each extremal monomorphism is an equalizer.

If $\mathfrak{A}$ is a subcategory of $\mathfrak{E}$, then the following are equivalent:

(1) $\mathfrak{A}$ is epi-reflective in $\mathfrak{E}$.

(2) $\mathfrak{A}$ is strongly closed under limits. 
(3) $\mathfrak{A}$ is strongly closed under products $\left({ }^{8}\right)$ and pullbacks.

(4) $\mathfrak{A}$ is strongly closed under products and inverse images.

(5) $\mathfrak{A}$ is strongly closed under products and finite intersections.

(6) $\mathfrak{A}$ is strongly closed under products and intersections.

(7) $\mathfrak{A}$ is strongly closed under products and equalizers.

(8) $\mathfrak{A}$ is strongly closed under products and extremal subobjects.

Proof. We will show that $(1) \Rightarrow(2) \Rightarrow(3) \Rightarrow(4) \Rightarrow(5) \Rightarrow(7) \Rightarrow(8) \Rightarrow(1)$ and (2) $\Rightarrow(6) \Rightarrow(5)$.

By Theorem 7, (1) $\Rightarrow(2)$; since products and pullbacks are limits, $(2) \Rightarrow(3)$; since inverse images are pullbacks, $(3) \Rightarrow(4)$; and since finite intersections are inverse images, (4) $\Rightarrow(5)$. By (iv), (7) $\Rightarrow(8)$, and by Theorem $6,(8) \Rightarrow(1)$. Since all intersections are limits, $(2) \Rightarrow(6)$, and $(6) \Rightarrow(5)$ trivially. Thus it remains to be shown that $(5) \Rightarrow(7)$.

Suppose that $\mathfrak{A}$ is strongly closed under products and finite intersections and that $f, g: A \rightarrow X$ are morphisms in $\mathfrak{E}$, where $A$ is an object of $\mathfrak{A}$. We wish to show that the equalizer of $f$ and $g$ is in $\mathfrak{A}$. Let $\left\{A \times X, p_{A}, p_{X}\right\}$ be a product with its projection morphisms. Let $\tilde{f}, \tilde{g}: A \rightarrow A \times X$ be the morphisms determined by the morphism pairs $\left(1_{A}, f\right)$ and $\left(1_{A}, g\right)$, respectively.

Let $h$ and $k$ be morphisms such that $\tilde{f} h=\tilde{f} k$. Then $p_{A} \tilde{f} h=p_{A} \tilde{f} k$, so that $1_{A} h=1_{A} k$. Hence $\tilde{f}$ is a monomorphism. Likewise $\tilde{g}$ is a monomorphism, so that since $\mathfrak{A}$ is strongly closed under finite intersections, the intersection $(Q, \alpha, \beta)$ is such that $Q$ is in $\mathfrak{A}$ and such that the diagram

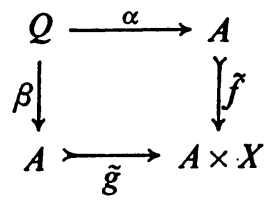

commutes. Thus $\tilde{f} \alpha=\tilde{g} \beta$ so that

$$
\alpha=1_{A} \alpha=p_{A} \tilde{f} \alpha=p_{A} \tilde{g} \beta=1_{A} \beta=\beta .
$$

Hence

$$
g \alpha=\left(p_{X} \tilde{g}\right) \alpha=p_{X}(\tilde{g} \alpha)=p_{X}(\tilde{f} \alpha)=\left(p_{X} \tilde{f}\right) \alpha=f \alpha
$$

so that $(Q, \alpha)$ is a lower bound of the diagram

$$
A \underset{g}{\stackrel{f}{\longrightarrow}} X .
$$

$\left.{ }^{8}\right)$ Note that by Definition 10, to be strongly closed under products is equivalent to being closed under products. 
Suppose that $E \stackrel{u}{\rightarrow} A$ is such that $f u=g u$. Then $\tilde{f u}=\tilde{g} u$ so that there exists a unique map $s: E \rightarrow Q$ such that the diagram

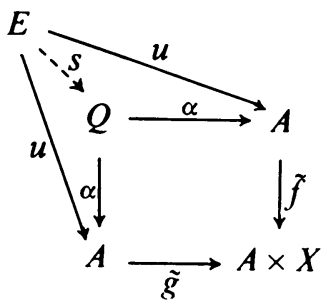

commutes. Consequently, $(Q, \alpha)$ is an equalizer for $f$ and $g$.

REMARK. In the above theorem, note that if the word "strongly" is left out, (2), (3), (4), (5), (6) and (7) remain equivalent. However in this form they do not characterize epi-reflections.

6. Generation of mono-coreflective subcategories. The following proposition is an immediate consequence of Theorem 6.

Proposition 6. If $\mathfrak{C}$ is a category which

(i) is locally small,

(ii) has coproducts,

(iii) has the extremal epi-mono factorization property;

then the intersection of any collection of mono-coreflective subcategories of $\mathbb{C}$ is a mono-coreflective subcategory of $\mathfrak{E}$.

LEMMA 4. Let $\mathbb{E}$ be any category with the strong unique extremal epi-mono factorization property. Then if $\left\{S_{i} \stackrel{u_{i}}{\rightarrow} S\right\}$ and $\left\{X_{i} \stackrel{v_{i}}{\rightarrow} X\right\}$ are coproducts in $\sqrt{ }$ and if for each $i$ there exists an extremal epimorphism $e_{i}: S_{i} \rightarrow X_{i}$, then the uniquely determined morphism $e: S \rightarrow X$ which for each $i$ makes the diagram

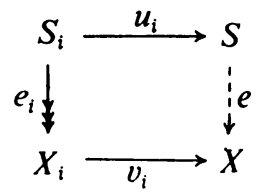

commute is an extremal epimorphism.

Proof. Let $p$ and $q$ be morphisms such that $p e=q e$. Then for each $i, p e u_{i}=q e u_{i}$, so that $p v_{i} e_{i}=q v_{i} e_{i}$. But each $e_{i}$ is an epimorphism, so that we have $p v_{i}=q v_{i}$ for each $i$. Thus by the definition of coproduct $p=q$. Hence $e$ is an epimorphism.

To show that $e$ is extremal, we let $g f$ be a factorization of $e$, where $g$ is a monomorphism. Then by Proposition 5, for each $i$ there exists a morphism $w_{i}$ which makes the diagram 


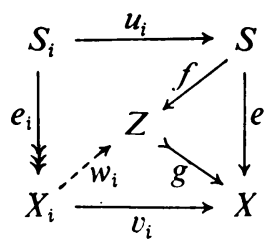

commute. Thus by the definition of coproduct, there exists a morphism $h: X \rightarrow Z$ such that for each $i, w_{i}=h v_{i}$. Hence for each $i, g h v_{i}=g w_{i}=v_{i}=1_{X} v_{i}$, so that $g h=1_{X}$. Therefore $g$ is a monomorphism and a retraction, so that it is an isomorphism.

Proposition 7. If 〔 is a category which

(i) is locally small,

(ii) has coproducts,

(iii) has the extremal epi-mono factorization property,

and if $\mathfrak{A}$ is a subcategory of $\mathfrak{C}$; then there exists a smallest mono-coreflective subcategory, $\mathfrak{B}$, of $\mathfrak{C}$ containing $\mathfrak{A}$. Furthermore if $\mathfrak{C}$ has the strong unique extremal epimono factorization property, then the objects of $\mathfrak{B}$ are exactly all extremal quotient objects of coproducts of objects in $\mathfrak{A}$.

Proof. Since via the identity functor, $\mathfrak{C}$ is mono-coreflective in itself, the first conclusion follows immediately from Proposition 6 . Let $\mathfrak{D}$ be the subcategory of $\mathfrak{A}$ whose objects are all the extremal quotient objects of coproducts of objects in $\mathfrak{A}$. To prove the second conclusion of the proposition, we must show that $\mathfrak{D}=\mathfrak{B}$. By Theorem 6 it is clear that $\mathfrak{D}$ is a subcategory of $\mathfrak{B}$ and that to show that $\mathfrak{B}$ is a subcategory of $\mathfrak{D}$ it is sufficient to show that $\mathfrak{D}$ is closed under the formation of coproducts and extremal quotient objects. By Definition 6(iii), $\mathfrak{D}$ is closed under the formation of extremal quotient objects. To show that it is closed under the formation of coproducts, let $\left\{X_{i} \stackrel{v_{i}}{\rightarrow} X\right\}$ be a coproduct where each $X_{i}$ is in $\mathscr{D}$. By the construction of $\mathfrak{D}$, for each $i$ there exists an object $S_{i}$ and an extremal epimorphism $q_{i}: S_{i} \rightarrow X_{i}$, where each $S_{i}$ is the object of a coproduct $\left\{S_{j}^{i} \stackrel{u_{j}^{i}}{\rightarrow} S_{i}\right\}$ where each $S_{j}^{i}$ is in $\mathfrak{A}$. Let $\left\{S_{j}^{i} \stackrel{w_{j}^{i}}{\rightarrow} S\right\}$ be a coproduct of all of the $S_{j}^{i}$. By the definition of coproduct, for each $i$ there exists a morphism $f_{i}: S_{i} \rightarrow S$ such that the diagram

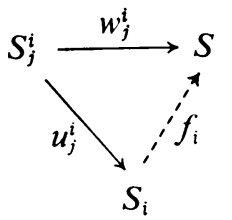

commutes. Hence $\left\{S_{i} \stackrel{f_{i}}{\rightarrow} S\right\}$ is a coproduct and by Lemma 4, the unique morphism $e: S \rightarrow X$ which makes the diagram

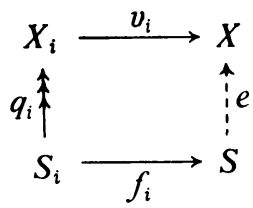


commute is an extremal epimorphism. Thus $X$ is an extremal quotient object of a sum of objects in $\mathfrak{A}$, so that $X$ is in $\mathfrak{D}$.

Notation. We denote the smallest mono-coreflective subcategory of $\mathfrak{s}$ which contains $\mathfrak{A}$ by $\mathfrak{S}(\mathfrak{U})$.

DeFINITION 11. If $\mathfrak{A}$ is a subcategory of a category, $\mathfrak{E}$, then a morphism $f: X \rightarrow Y$ in $\mathfrak{C}$ is said to be $\mathfrak{A}$-liftable provided that for each object $A$ in $\mathfrak{A}$ and each morphism $g: A \rightarrow Y$, there exists a unique morphism $g^{\prime}: A \rightarrow X$ such that $g=f g^{\prime}$.

THEOREM 9. If $\mathbb{E}$ is a category which

(i) is locally small,

(ii) has coproducts,

(iii) has the strong unique extremal epi-mono factorization property, and if $\mathfrak{A}$ is any subcategory of $\mathfrak{E}$, then each monomorphism in $\mathfrak{\mathbb { N }}$ which is $\mathfrak{A}$-liftable is also $\mathfrak{C}(\mathfrak{U})$-liftable.

Proof. Let $f: X \rightarrow Y$ be the given monomorphism. Let $B$ be an object of $\mathfrak{S}(\mathfrak{U})$ and let $g: B \rightarrow Y$. By Proposition 7 there exists a coproduct $\left\{A_{i} \stackrel{u_{i}}{\rightarrow} S\right\}$ of objects of $\mathfrak{A}$ and an extremal epimorphism $h: S \rightarrow B$. Now for each $i, g h u_{i}: A_{i} \rightarrow Y$, so that by the hypothesis there exists a unique morphism $v_{i}: A_{i} \rightarrow X$ such that $f v_{i}=g h u_{i}$. Thus by the definition of coproduct, there exists a unique morphism $v: S \rightarrow X$ such that for each $i, v_{i}=v u_{i}$. Finally, by Proposition 5 , there exists a unique morphism $g^{\prime}: B \rightarrow X$ such that the diagram

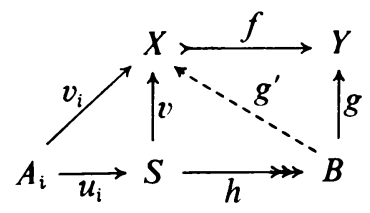

commutes. Thus $f$ is $\mathfrak{C}(\mathfrak{A})$-liftable.

COROLlaRY. Let $\mathfrak{c}$ be a category which

(i) is locally small,

(ii) has coproducts,

(iii) has the strong unique extremal epi-mono factorization property.

Then if $\mathfrak{A}$ is a subcategory of $\mathfrak{E}, X$ an object of $\mathfrak{E}(\mathfrak{A}), f: X \rightarrow Y$ any $\mathfrak{A}$-liftable monomorphism, and $c_{\mathfrak{C}(\mathfrak{H})}: Y_{\mathfrak{C}(\mathfrak{I})} \rightarrow Y$ the coreflection morphism for $Y$, then $f: X \rightarrow Y$ and $c_{\mathfrak{C}(\mathfrak{I})}: Y_{\mathfrak{C}(\mathfrak{A})} \rightarrow Y$ are isomorphic subobjects so that $f$ is essentially the coreflection $c_{\mathfrak{E}(\mathfrak{Q})}$.

\section{REFERENCES}

1. S. Baron, Reflective subcategories of colocally small categories, Notices Amer. Math. Soc. 13 (1966), 609. Abstract \#636-112.

2. - Reflective subcategories of colocally small (cowell powered) categories. II, Notices Amer. Math. Soc. 14 (1967), 516-517. Abstract \#67T-331. 
3. N. Bourbaki, Algèbre, Actualités Sci. Indust., no. 1044, Hermann, Paris, 1948. MR 10, 231.

4. E. Cech, On bicompact spaces, Ann. of Math. (2) 38 (1937), 823-844.

5. P. Freyd, Abelian categories. An introduction to the theory of functors, Harper's Series in Modern Math., Harper \& Row, New York, 1964. MR 29 \#3517.

6. A. M. Gleason, Universal locally connected refinements, Illinois J. Math. 7 (1963), 521-531. MR 29 \#1612.

7. H. Herrlich, Contributions to extension theory of topological structures, VEB Deutscher Verlag der Wissenschaften, Berlin, 1969, pp. 105-114.

8. - Constant maps in categories (to appear).

9. H. Herrlich and J. van der Slot, Properties which are closely related to compactness, Nederl. Akad. Wetensch. Proc. Ser. A 70=Indag. Math. 29 (1967), 524-529. MR 36 \#5898.

10. P. J. Hilton, Catégories non-Abéliennes, Lecture Notes, University of Montreal, 1964.

11. M. Hušek, Remarks on reflections, Comment. Math. Univ. Carolinae 7 (1966), 249-259. MR 34 \#2659.

12. — One more remark on reflections, Comment. Math. Univ. Carolinae 8 (1967), 129-137. MR 35 \#232.

13. J. R. Isbell, Some remarks concerning categories and subspaces, Canad. J. Math. 9 (1957), 563-577. MR 20 \#923.

14. —_, Natural sums and abelianizing, Pacific J. Math. 14 (1964), 1265-1281. MR 31 \#3478.

15. - Subobjects, adequacy, completeness and categories of algebras, Rozprawy Mat. 36 (1964). MR 29 \#1238.

16. — Structure of categories, Bull. Amer. Math. Soc. 72 (1966), 619-655. MR 34 \#5896.

17. J. F. Kennison, Reflective functors in general topology and elsewhere, Trans. Amer. Math. Soc. 118 (1965), 303-315. MR 30 \#4812.

18. — Full reflective subcategories and generalized covering spaces, Illinois J. Math. 12 (1968), 353-365. MR 37 \#2832.

19. B. Mitchell, Theory of categories, Pure and Appl. Math., vol. 17, Academic Press, New York, 1965. MR 34 \#2647.

Freie UNIVERsität BerLin, Berlin-Dahlem, Federal Republic of Germany UNIVERSITY OF FLORIDA, GAINESVILLE, FLORIDA 32601 